\title{
Liquid crystal tunable filters in detecting water pollution
}

\author{
Damian Wierzbicki, Michalina Wilińska \\ Department of Remote Sensing and Photogrammetry, Institute of Geodesy, Faculty of Civil Engineering and Geodesy, \\ Military University of Technology, 2 gen. Sylwestra Kaliskiego st., 00-908 Warsaw, Poland
}

\begin{abstract}
In this article a methodology for determining the transmission of two electronically tunable interference filters Varispec CRI-VIS and CRI-SNIR had been described. The aim of the research was to determine the filter transmission values and to compare them with the results provided by the filter's producer. The tested interference filters are built with the intent of being integrated with panchromatic video cameras (400-1100 nm). As a result of this integration, hyperspectral sensors can be created. The acquired data could then be used to determine spectral reflectance characteristics, and on that basis, a methodology of the detection of physical, chemical and biological pollutants in rivers, coastal zones and flood areas will be elaborated. The precise determination of the filters transmission values is necessary to establish the correct exposure parameters. Thanks to a correct methodology of data acquisition, the collected images could be used for qualitative and quantitative analyses of surface water pollutants. Within the framework of the presented research, laboratory measurements of the filter transmissions were carried out for the CRI-SNIR-10-20 filter (for the interval from $650 \mathrm{~nm}$ to $1100 \mathrm{~nm}$ ) and the CRI-VIS-20-20 filter (for the interval from $400 \mathrm{~nm}$ to $720 \mathrm{~nm}$ ). The main concept of the research was to calibrate a spectroradiometer using a reference panel and then to perform a series of measurements of the light transmitted through the filter which had been tuned to a specific wavelength. The measurements were conducted using Field Spec 3, Field Spec 4 Wide-Res spectroradiometers and a JASCO V670 spectrophotometer. In addition, using a goniometer, an analysis of the influence of the measurement station geometry on the filters transmission values were carried out.
\end{abstract}

Keywords: environmental protection; water engineering; Liquid crystal tunable filters (LCTF); transmission; water pollution.

\begin{tabular}{|ll|}
\hline \multicolumn{2}{|l|}{ Nomenclature } \\
$\mathrm{n}$ & refractive index \\
$\mathrm{d}$ & the thickness of the filter plate \\
$\mathrm{T}$ & transmission value $(\%)$ \\
Greek & symbols \\
A & illumination angle \\
$\lambda$ & wavelength (nm) \\
$\delta$ & the phase delay \\
\hline
\end{tabular}

\section{Introduction}

For several years, we have been able to observe a growing interest in the search for innovative remote sensing methods for monitoring the state of pollution of inland waters using hyperspectral imaging [1], [2], [6], [9], [11], [18], [19]. To date, in order to determine the various parameters of physical, biological and chemical properties of water, it was common to use multi- and hyperspectral data acquired from satellite imaging systems (ALI/EO-1, CHRIS/PROBA, ASTER/TERRA, HYPERION/EO-1, Landsat ETM+) [5], [7], [10], [13-16], [20]. When using these research methods, the value of the ground sampling distance, which is often from ten to hundreds of meters, is primarily cause for restrictions. Therefore, it is possible to perform a rough estimate of the water quality only for large water basins. In addition, the most complicated process is often the search for relationships between the acquired satellite data and the values of water indicators measured directly in the field. Therefore, in situ measurements are a much more reliable research method. With respect to researching water quality and pollution, optical methods relying on a function of reflection of incident light in relation to the depth of

Corresponding author: Michalina Wilińska. E-mail address: mwilinska@wat.edu.pl

http://dx.doi.org/10.3846/enviro.2014.013

(C) 2014 The Authors. Published by VGTU Press. This is an open-access article distributed under the terms of the Creative Commons Attribution License, which permits unrestricted use, distribution, and reproduction in any medium, provided the original author and source are credited. 
the water reservoir are mostly used. It is possible to determine what part of the light spectrum penetrating the water depth is absorbed by the optically active components on the basis of the measured reflectance spectrum of illumination on the water surface [4]. While determining the value of the water reflectance factor [12] it is known, that the incident light beam is reflected in all directions. However, optical sensors onboard of remote sensing satellites are designed for capturing radiant flux from a particular direction, so the reflectance only from a small solid angle in a particular direction is registered which causes considerable uncertainty in the results.

The paper presents a methodology for determining the transmission $\mathrm{T}$ of electronically tunable liquid crystal filters. At the time of the research, the impact of the light incidence angle on the obtained values of the filters' transmissions was included. The developed methodology for determining the transmission of such filters, in the future will allow us to use them in in situ measurements for determining inland water depths, and thus to indirectly detect certain water quality indicators. In the future, the research will focus on the detection of specific physicochemical and biological contaminants occurring in inland waters.

\section{Construction and operation principles of the tested LCTF}

In our research, two electronically tunable liquid crystal filters - CRI-VIS-20-20 and CRI-SNIR-10-20 were used, with EM radiation ranges from $400 \mathrm{~nm}$ to $720 \mathrm{~nm}$ and from $650 \mathrm{~nm}$ to $1100 \mathrm{~nm}$ respectively [24]. The operational principle of LCTFs is similar to high-quality interference filters, except that the wavelength of the light which is transmitted is electronically controlled to assure prompt and a vibration-free selection of any wavelength within a specified range of the spectrum. They operate by using two phenomena: polarization [8], [21] and birefringence [23], [25]. Generally, the VariSpec CRI-VIS-2020 and CRI-SNIR-10-20 filters are multilayer Lyot-Ohman type interference filters, but with a liquid crystal surface (called a waveplate) in each layer, which allows us to electronically control the corresponding difference in phase of the wave surface. The filter includes optical linear polarizers, a layer of quartz and liquid crystal elements. Liquid crystal components used in the filter allow us to tune the operating range of the filter by applying an electrical voltage to the liquid crystal, which changes the angle of plane polarization of the passing light. More details on the principle of their operation is shown in [22]. Each polarizer introduces a transmission loss of about 5\%, resulting in a total loss of transmission which can be as high as $50-70 \%$ [17]. It should be noted, that in the case, where the incident light is polarized then the transmission may increase up to double compared to randomly polarized light [26]. The bandwidth which is passed through the filter is defined by the width at half height of the transmission curve - FWHM - i.e. the number of adjacent wavelength carried in FWHM, besides the wavelengths tuned at the moment. For the Lyot filters, full width at half maximum is constant and changes every $1 / 8$ of the wavelength [24]. The considered VariSpec liquid crystal filters can retune every $1 \mathrm{~nm}$, however, it does not mean, that the filter passes such a narrow range of wavelength. In reality it is a bandwidth of 7, $10 \mathrm{or} 20 \mathrm{~nm}$, depending on the filter model used. Filter transmission is a constant value, set at manufacturing and this parameter can't be changed by the user and it is defined as the spectral distance between two points, where filter transmission reaches $50 \%$ of the peak of the wave.

During the study of above mentioned VariSpec filters - CRI, the values of transmission had been determined. The values of transmission specified by the filters manufacturer for different wavelengths are shown in Figure 1.

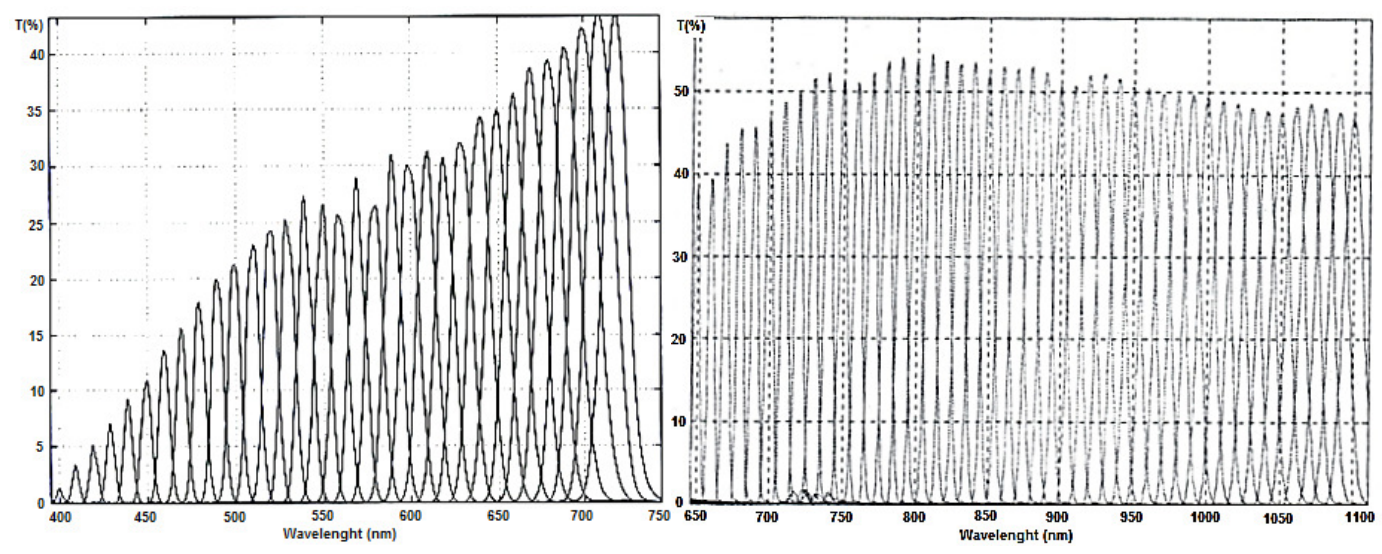

Fig. 1. The transmission of the studied filters CRI-VIS-20-20 (a) i CRI-SNIR-10-20 (b) - manufacturers data

The spectral transmission profile of liquid crystal filters is similar in shape to the Gaussian distribution function. In optical terms, the basics of retuning filters of this type were first formulated by Bernard Lyot in 1933. As is known, birefringent materials are characterized by different refractive indices $n_{0}$ and $n_{e}$ for two perpendicular polarization planes, which are usually defined as ordinary planes with extending along them ordinary rays. The difference $\Delta n=n_{0}-n_{e}$ determines the so-called phase delay between the entering and exiting radii from the filter. For birefringent plates with a $d$ thickness, the phase delay $\delta$ between the two radii is ( $\lambda$ - wavelength in vacuum): 


$$
\delta=\frac{2 \pi d \Delta n}{\lambda}
$$

In order to derive an equation for the value of the filters transmission a simple relationship between the amplitude value $A$ and the phase delay $\delta$ can be formulated:

$$
A_{\|}=A \cos (\delta)
$$

Because the transmission is defined as the ratio of the polarized light $I$ exiting from the filter to the light incident onto the filter, therefore between the amplitude and the light intensity there is the following relationship:

$$
I \infty A^{2}
$$

On the basis of Eqns (2) and (3) it can be stated, that the transmission value $T(\lambda)$ of a single layer of the Lyot liquid crystal filter can be expressed by the formula [3]:

$$
T(\lambda)=\cos ^{2}\left(\frac{2 \pi d \Delta n}{\lambda}\right)
$$

As seen in the above relationship, the filter transmission is dependent on wavelength - the shorter the wavelength, the transmission is smaller. This is a serious defect in the tested filters, because for the wavelength of $400 \mathrm{~nm}$ the transmission value is about 3\%. Such small light transmission causes significant difficulties in the use of these filters for image registration in selected spectral bands. This involves a considerable lengthening of the exposure times for transmission in the short wavelength ranges. This can cause a significant impact on the amount of detector dark current and also the effect of blurring while imaging from onboard an aircraft operating at considerable speeds.

\section{Characteristics of the measuring devices used for the determination of filter transmission}

During the VariSpec CRI filter tests, Field Spec 3, Field Spec 4 spectroradiometers and a JASCO V-670 spectrophotometer were used. Field Spec spectroradiometers are high-class devices, which perform measurements within the field of a single beam in the visible, near-and mid-infrared wavelength ranges. Spectroradiometers are equipped with sensors with a $1^{\circ}$ or $25^{\circ}$ field of view (FOV). The spectral resolution of the Field Spec 3 spectroradiometer is equal to $3 \mathrm{~nm}$ in the range of $\lambda$ from 350 to $1000 \mathrm{~nm}$ and $10 \mathrm{~nm}$ in the range of 1000 to $2500 \mathrm{~nm}$, whereas for Field Spec $4-30 \mathrm{~nm}$ in the range from 1000 to $2500 \mathrm{~nm}$. In this study, spectral data were generalized to intervals of $10 \mathrm{~nm}$. During the research, in addition to the spectroradiometers, a JASCO V670 spectrophotometer was also used. It measures the absorption spectrum from 190 to 2700 $\mathrm{nm}$, and the reflection spectrum in the range from 250 to $2500 \mathrm{~nm}$. In the range of 190 to $350 \mathrm{~nm}$ (UV) a deuterium lamp is used, and in the range of 330 to $2700 \mathrm{~nm}$ (visible and NIR), the spectrophotometer uses a halogen lamp. The principle of operation of the spectrophotometer and its technical data are shown in [27].

\section{The method of determining the VariSpec CRI filters transmission}

In the frame of research, the LCTF CRI-VIS-20-20 and CRI-SNIR-10-20 filters, both with a nominal bandwidth of $20 \mathrm{~nm}$, were studied. Measurements of the LCTF transmission were conducted in a laboratory, although it is also possible to test and verify them outside of a laboratory. These studies are extremely important from the point of view of future research in the "Innovative remote sensing system for the monitoring of pollutants in rivers, offshore waters and flooded areas" project. Knowledge of the filters transmission values is necessary to establish guidelines for future research concerning determining spectral characteristics of the most common physical, biological and chemical water pollutants. The first set of the laboratory tests were carried out using the Field Spec 3 spectroradiometer and then, controls measurements were obtained with the JASCO V670 spectrophotometer. The VariSpec filter CRI-VIS-20-20 was tested in a 400-720 nm range, while the CRI- SNIR-10-20 in 650-1100 nm.

The spectrophotometric laboratory measurement set consisted of a light source, white reflectance standard, a spectroradiometer and two interchangeably used LCTF filters. The white reference standard was positioned centrally relative to the pistol grip of the measuring device and was illuminated by a homogeneous non-polarized light, whose source was a halogen lamp. Before starting the measurement, the spectroradiometer was calibrated using the reference standard. Then the tested filters were mounted directly to the pistol grip. To prevent the scattering of the radiation, the space between the end of the pistol grip and the surface of the filter was sealed with a light-impermeable material. During the measurements collected spectral data were generalized to intervals $10 \mathrm{~nm}$, and for each spectrum 10 measuring series were performed. First, the filter was tuned electronically to a certain wavelength (in the range of 400 to $700 \mathrm{~nm}$ for CRI-VIS-2020 and from 650 to $1100 \mathrm{~nm}$ for CRI-SNIR-10-20 with $10 \mathrm{~nm}$ intervals), and next, after filter retuning, the received transmission was recorded using a portable computer. As a result of these measurements, the obtained data were used to plot graphs, which were in fact the tested filters transmission curves for a given wavelength (Fig. 2 and Fig. 3). 


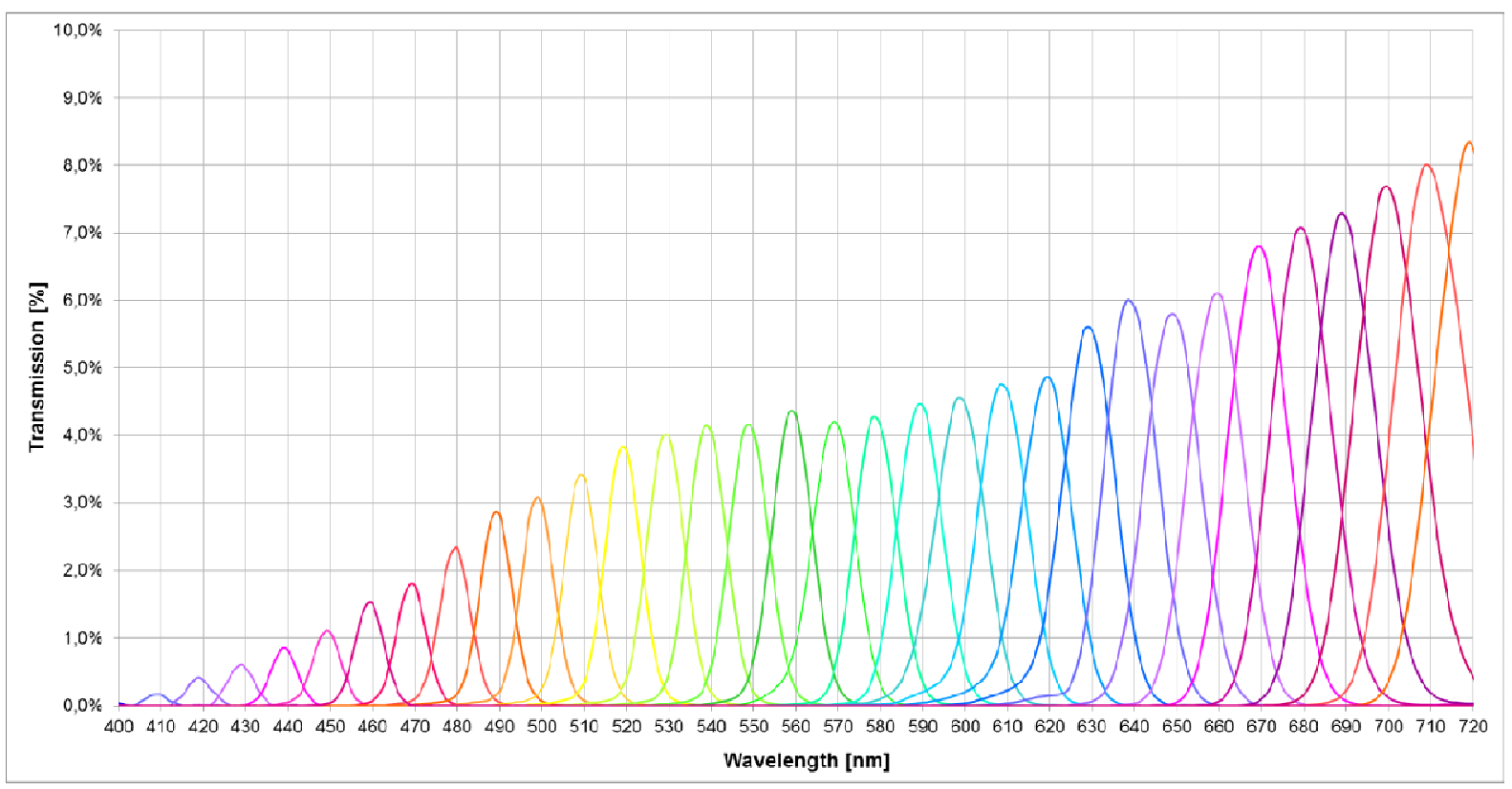

Fig. 2. VariSpec CRI-VIS-20-20 transmission values measured with Field Spec 3 spectroradiometer

Analyzing the filter transmission curves of the CRI-VIS-20-20 filter, we can conclude, that as expected, the transmission increases with increasing wavelength. The minimum value of the transmission was recorded at a wavelength of $400 \mathrm{~nm}$ and amounted to only $0.002 \%$. The maximum transmission value was reached at a wavelength of $720 \mathrm{~nm}$ and amounted to $8.3 \%$. Transmission values for wavelengths of 520-570 nm were at a similar level and its average value was $4.1 \%$. For the transmission of the CRI-SNIR-10-20 filter, it can be concluded that for $\lambda$ tuned every $10 \mathrm{~nm}$ in the range from $650 \mathrm{~nm}$ to $670 \mathrm{~nm}$ the transmission does not exceed the value $3.0 \%$, and for the $\lambda$ from $680 \mathrm{~nm}$ to $890 \mathrm{~nm}$ it reaches maximum values in the range from $4.3 \%$ to $5.6 \%$. It then gradually decreases, to $\lambda$ equal to $1050 \mathrm{~nm}, 1060 \mathrm{~nm}$ and $1070 \mathrm{~nm}$ achieving maximum transmission values below $0.4 \%$. For $\lambda$ equal to $1080 \mathrm{~nm}, 1090 \mathrm{~nm}$ and $1100 \mathrm{~nm}$ the transmission increases rapidly and reaches a maximum equal to $9.3 \%, 9.4 \%$ and $8.2 \%$ respectively.

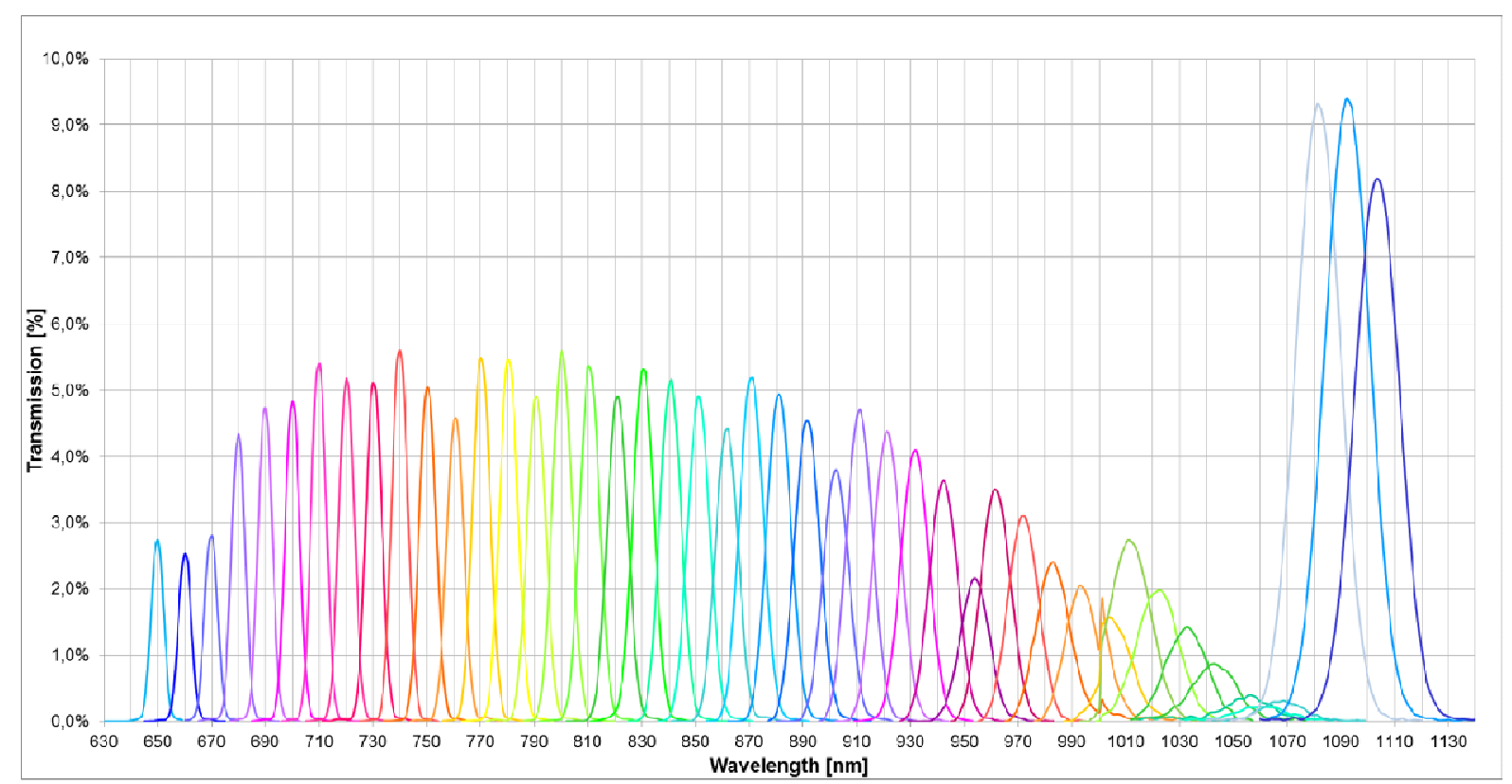

Fig. 3. VariSpec CRI-SNIR-10-20 transmission values measured with Field Spec 3 spectroradiometer

The results of transmission obtained for both filters differ significantly from the data presented by the manufacturer, so as a control measurement we tested them using a JASCO V670 spectrophotometer (Fig. 4 and Fig. 5). The transmission values were found to be noticeably higher in relation to the results obtained in studies conducted Field Spec 3 spectroradiometer. For the CRI-VIS-10-20 filter, the maximum recorded transmission value was achieved for $\lambda=719 \mathrm{~nm}$, and it amounted to $26.8 \%$. But for the CRI-SNIR-20-20 filter the transmission reaches its maximum for $\lambda=1080 \mathrm{~nm}$, and it was equal to $18.7 \%$. Similarly, as in the case of measurements performed with spectroradiometer, for some wavelengths $(\lambda=1050 \mathrm{~nm}$, $\lambda=1060 \mathrm{~nm}$ and $\lambda=1070 \mathrm{~nm}$ ) sharp declines can be observed in the transmission of the SNIR-CRI-20-20 filter. The 
recorded transmission curves generally do not depict any noise or sudden fluctuations in the transmission of other wavelengths than that which is being investigated at the moment. Although, values registered with a higher class equipment, such as the JASCO V670 spectrophotometer, were far greater than the values obtained with the Field Spec 3, they are still several fold lower than those presented by the manufacturer.

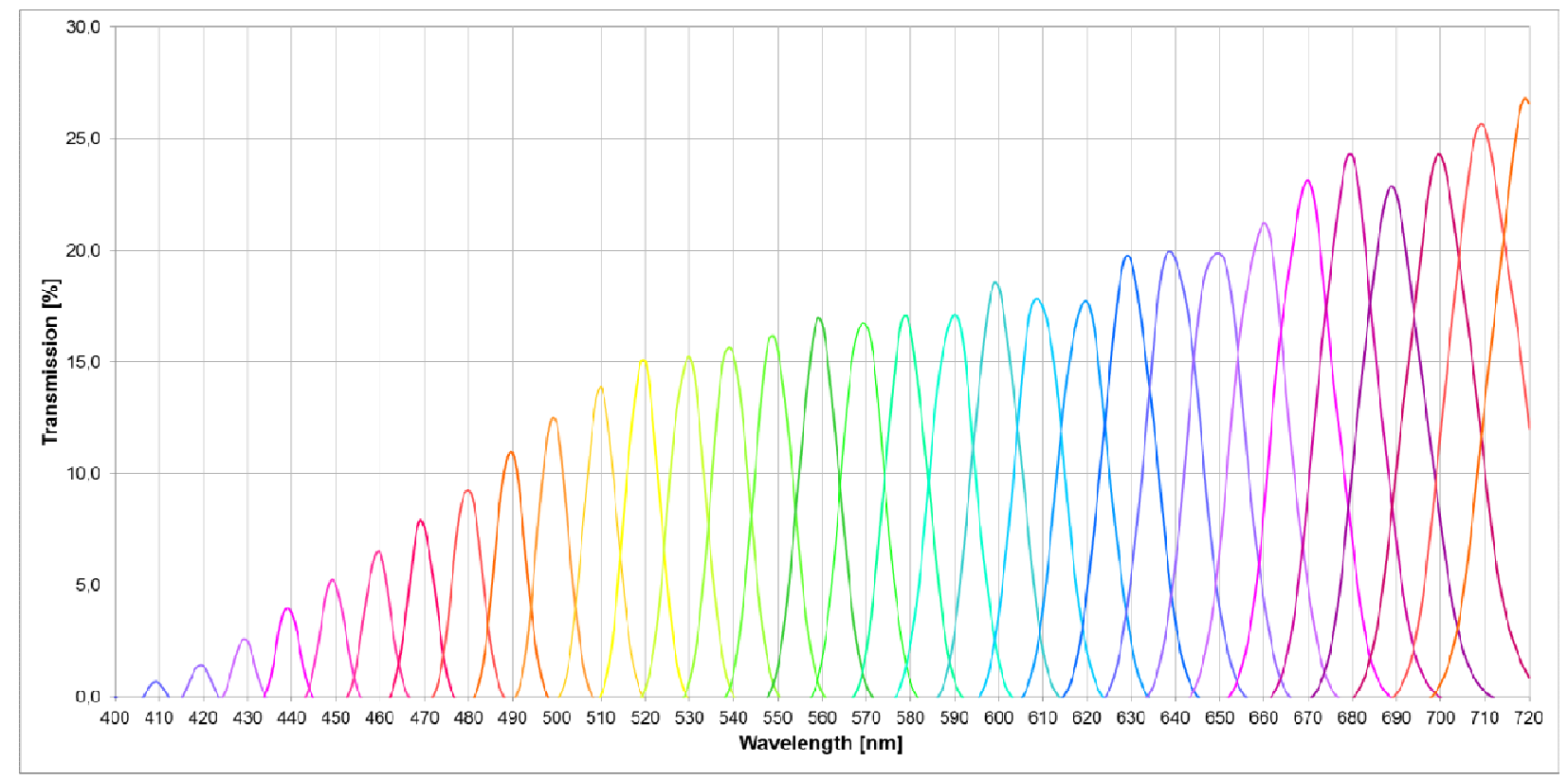

Fig. 4. VariSpec CRI-VIS-20-20 transmission values measured with JASCO V670 spectrophotometer

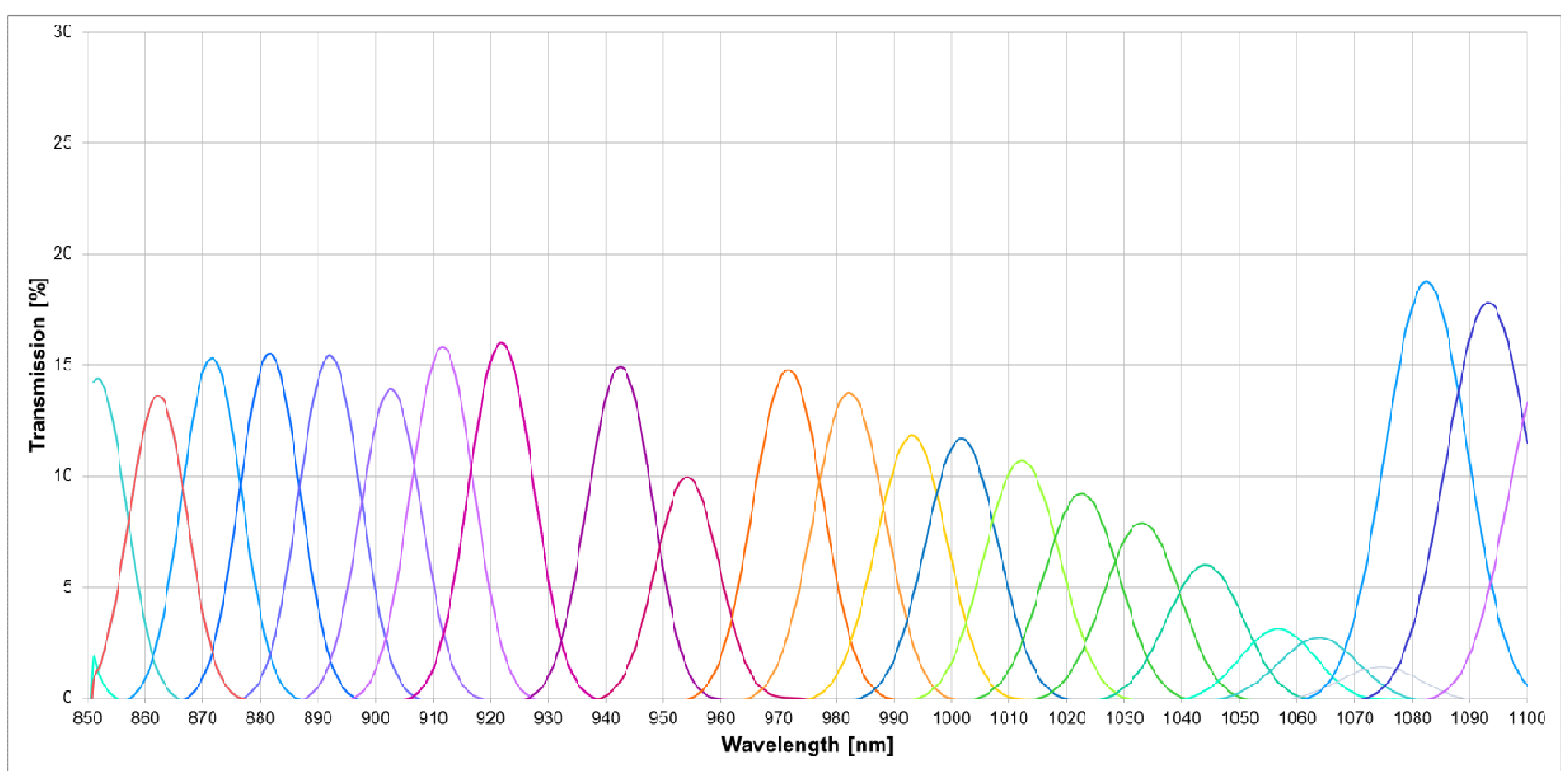

Fig. 5. VariSpec CRI-SNIR-10-20 transmission values measured with JASCO V670 spectrophotometer

\section{CRI-VIS-10-20 and CRI-SNIR-20-20 filters transmission values as a function of the illumination angle $\alpha$}

The purpose of this part of the research, was to develop methods and to assess the studied filters transmission variability as a function of illumination angle $\alpha$. For this purpose a special goniometer was designed, so that it was possible to adjust the light incidence angle relative to the reflective pattern with an interval equal to $10^{\circ}$ in the range from $10^{\circ}$ to $80^{\circ}$. The research scheme during this measurement procedure was the same as in the previous case, except that this time the Field Spec 4 Wide Res spectroradiometer was used. The measurement results are shown in Figures 6 and 7. 


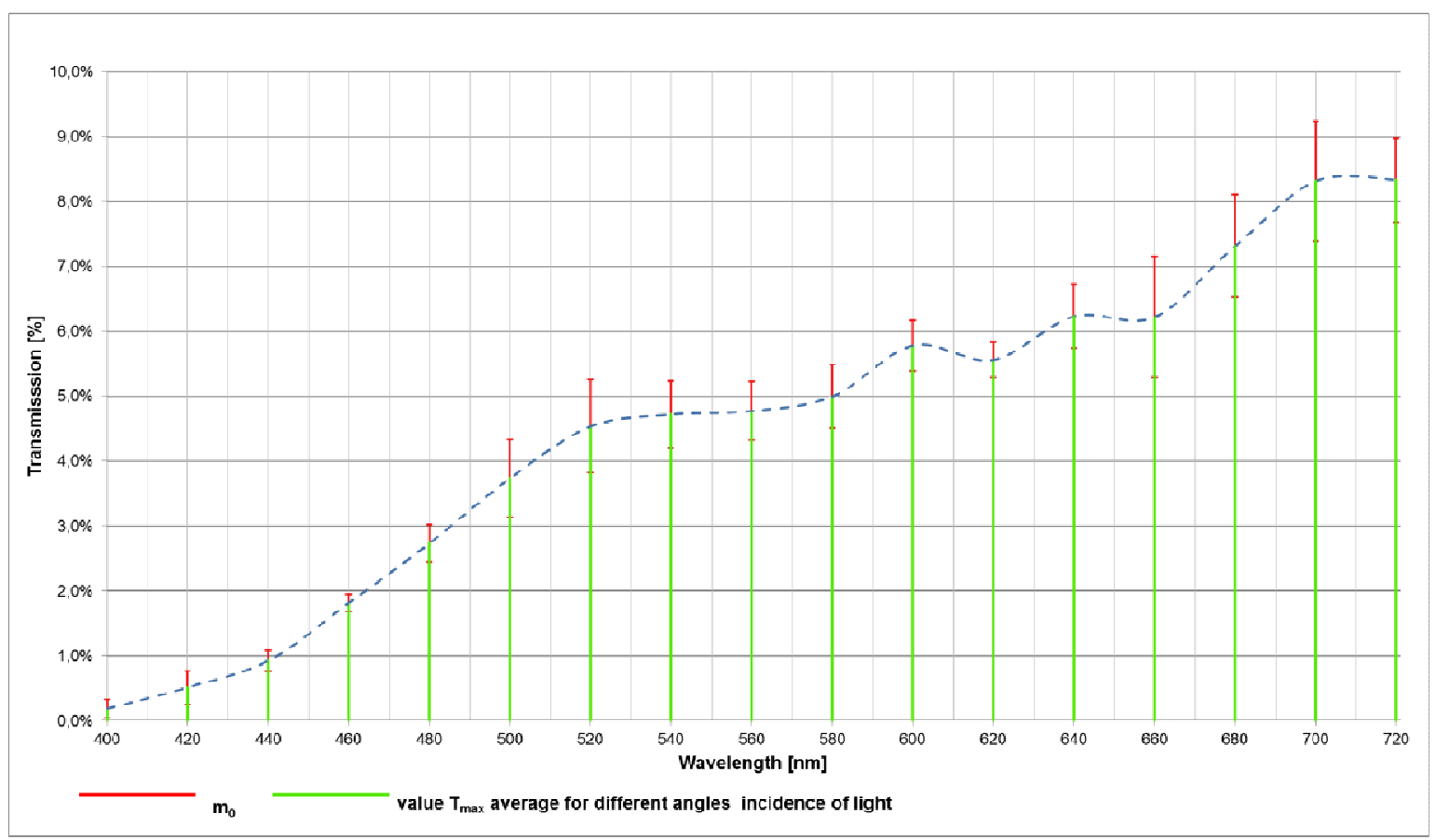

Fig. 6. The maximum values $T_{\max }$ of the filter transmission LCTF VariSpec VIS averaged for different light incidence angles with the distribution of the mean errors

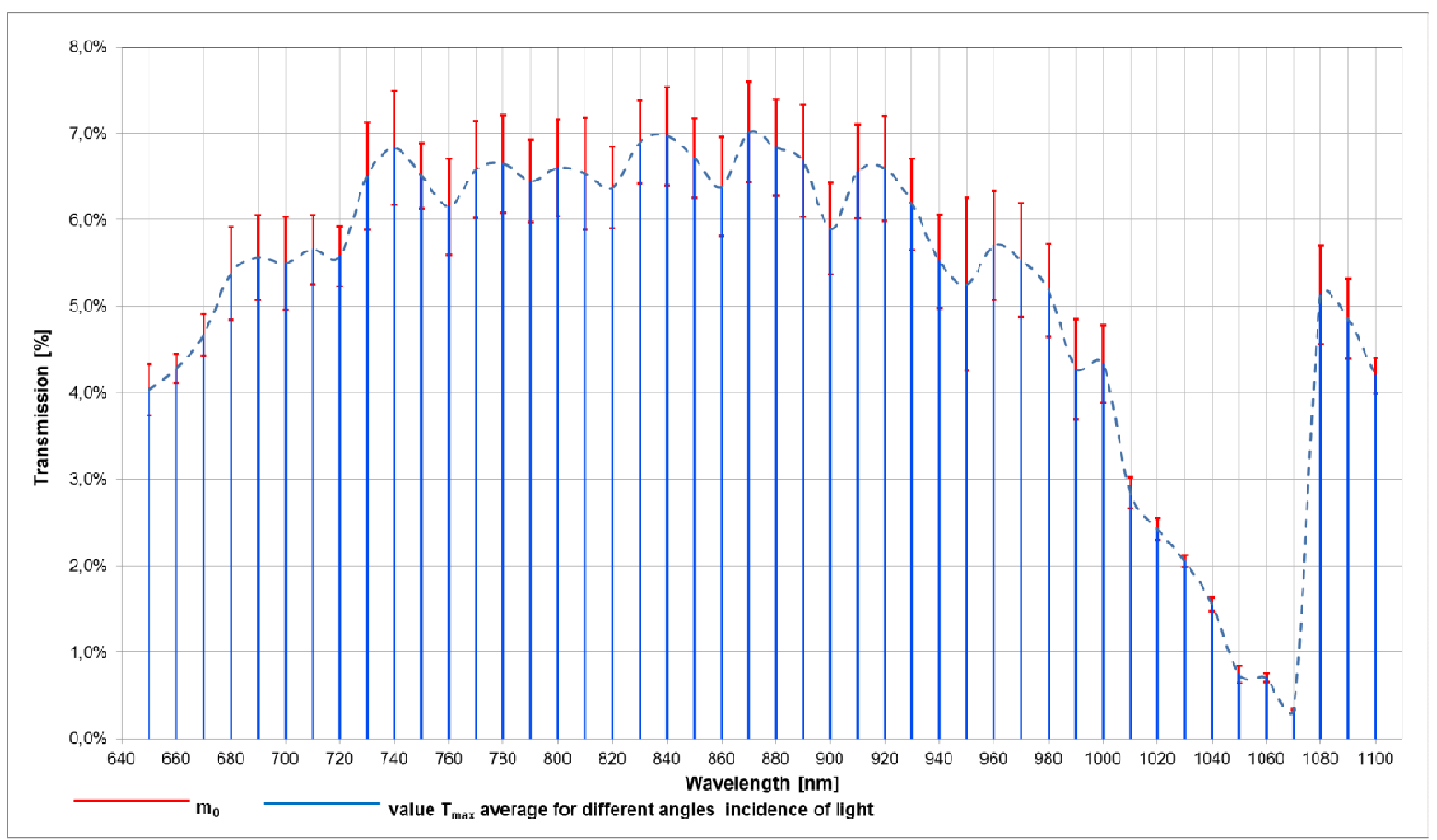

Fig. 7. The maximum values $\mathrm{T}_{\max }$ of the filter transmission LCTF VariSpec SNIR averaged for different light incidence angles with the distribution of the mean errors

On the basis of the conducted studies, it can be concluded, that the transmission values of the VariSpec filters vary depending on the lighting angle $\alpha$. The standard deviation of the maximum of transmissions is on average $0.45 \%$ for CRISNIR-10-20 and 0.49\% for CRI-VIS-20-20, which is within the measurement margin of error. On the basis of the test results, it can't be clearly determined for which illumination angle $\alpha$, we achieve the highest transmission values for all $\lambda$, and at which it is the lowest. However, the closer the lighting angles of the white reference panel were to $90^{\circ}$, the higher the transmission. As noted in previous studies, due to the fact, that the tested filter operates based on light polarization, our research had confirmed that the obtained filter transmission values are dependent on the incidence angle of the light. However, the values by which the individual results differ, are of the order of the measurement error, and therefore they can be considered negligible. 


\section{The method of determining the VariSpec CRI filters transmission}

As part of this research, laboratory measurements were made of the transmission of the CRI-VIS-20-20 filter in the $400 \mathrm{~nm}$ to $720 \mathrm{~nm}$ range and of the CRI-SNIR-10-20 filter in the $650 \mathrm{~nm}$ to $1100 \mathrm{~nm}$ range in steps of $10 \mathrm{~nm}$. Analyzing the results of transmission measurements obtained by three independent devices, we can conclude, that they are almost 10 times lower than the values given by the manufacturer. The reason for this may be that during laboratory work the filter was illuminated with a halogen lamp, whose light is scattered and isn't polarized. Whereas filters tested by the manufacturer, probably already at the input of the filter, were illuminated by polarized light, and the light coming out of them were re-polarized, which gave transmission values in excess of $50 \%$. Based on the study's results of the influence of illumination angle on the transmission values, it can be said, that it affects the transmission of VariSpec CRI-SNIR and CRI-VIS filters, but the differences are within the measurement error and can be considered as negligible. Overall, the data provided by the manufacturer may not be possible to achieve in natural measurement conditions, especially in the case when it comes to the use of a filter CRI-SNIR-10-20 for recording the water surface and analyzing its contaminant. As a result of above, it had been observed that these filters will be adequate in imaging studies using a microscope etc., but when registering images of the Earth's surface, it is necessary to take into account the filter transmission determined using a spectroradiometer (to respectively increase exposure time). In terms of the project IRAMS, designated tested filters transmission parameters will be particularly important in the image acquisition in laboratory conditions, and therefore it was necessary to make a special test station, which is the subject of other research.

\section{Acknowledgments}

The work described in this paper had been conducted as part of the "IRAMSWater - Innovative remote sensing system for the monitoring of pollutants in rivers, offshore waters and flooded areas" project (PBS1/B9/8/2012). The project is financed by the polish National Centre for Research and Development.

\section{References}

[1] Abdulhalim, I. 2009. Optimized guided mode resonant structure as thermooptic sensor and liquid crystal tunable filter, Chinese. Optics Letters 7: 667670 .

[2] Álvarez-Romero, J. G.; Devlin, M. J.; Teixeira da Silva, E.; Petus, C.; Ban, N.; Pressey, R. J.; Kool, J.; Roberts, S.; Cerdeira, W. A.; Brodie, J. 2013. A novel approach to model exposure of coastal-marine ecosystems to riverine flood plumes based on remote sensing techniques, J Environ Manage 119 : 194-207. http://dx.doi.org/10.1016/j.jenvman.2013.01.036

[3] Brettel, H.; Hardeberg, J. Y.; Schmitt, F. 1999. Multispectral Image Capture Across the Web, in The Seventh Color Imaging Conference: Color Science, Systems, and Applications, pages 314-316, Scottsdale, AZ, 314-316.

[4] Ficek, D. 2013. The bio-optical properties of waters in Pomeranian lakes (Poland) and their comparison with the properties of waters in other lakes and the Baltic Sea. Diss. Monogr. No. 23, 351 pp.

[5] Flink, P.; Lindell, T.; Ostlund, C. 2001. Statistical analysis of hyperspectral data from two Swedish lakes, The Science of the Total Environment 268: 155-169. http://dx.doi.org/10.1016/S0048-9697(00)00686-0

[6] Graça, M. A.; Rodrígues-Capítulo, A.; Ocón, C.; Gómez, N. 2002. In situ tests for water quality assessment: a case study in Pampean rivers, Water research 36(16): 4033-4040. http://dx.doi.org/10.1016/S0043-1354(02)00132-X

[7] Hellweger, F. L.; Schlosser, P.; Lall, U.; Weissel, J. K. 2004. Use of satellite imagery for water quality studies in New York Harbor, Estuarine Coastal and Shelf Science 61(3): 437-448. http://dx.doi.org/10.1016/j.ecss.2004.06.019

[8] Hensley, B.; Wyble, D. 2012. Spectral Imaging Using a Liquid Crystal Tunable Filter, [online] Available from Internet: http://www.cis.rit.edu/DocumentLibrary/admin/uploads/CIS000196.pdf

[9] Homma, K.; Shibayama, M.; Yamamoto, H.; Sugahara, K.; Shingu, H. 2005. Water pollution monitoring using a hyperspectral imaging spectropolarimeter, in Fourth International Asia-Pacific Environmental Remote Sensing Symposium 2004: Remote Sensing of the Atmosphere, Ocean, Environment, and Space (pp. 419-426). International Society for Optics and Photonics.

[10] Hu, C.; Chen, Z.; Clayton, T. D.; Swarzenski, P.; Brock, J. C.; Muller-Karger, F. E. 2004. Assessment of estuarine water-quality indicators using MODIS medium-resolution bands: Initial results from Tampa Bay, FL, Remote Sensing of Environment 93(3): 423-441. http://dx.doi.org/10.1016/j.rse.2004.08.007

[11] Huang, G. H.; Xia, J. 2001. Barriers to sustainable water-quality management, Journal of Environmental Management. http://dx.doi.org/10.1006/jema.2000.0394

[12] Kaminski, P.; Walczykowski, P.; Orych, A. 2013. Accuracy of determining spectral reflectance coefficients in a function of time, in Conference Materials, Virtual Research Conference ARSA - Advanced Research in Scientific Areas

[13] Kavurmaci, M.; Ekercin, S.; Altaş, L.; Kurmaç, Y. 2013. Use of EO-1 Advanced Land Imager (ALI) multispectral image data and real-time field sampling for water quality mapping in the Hirfanlı Dam Lake, Turkey, Environmental Science and Pollution Research, 1-9.

[14] Kloiber, S. M.; Brezonik, P. L.; Olmanson, L. G.; Bauer, M. E. 2002. A procedure for regional lake water clarity assessment using Landsat multispectral data, Remote Sensing of Environment 82: 38-47. http://dx.doi.org/10.1016/S0034-4257(02)00022-6

[15] Li, S. J.; Wang, X. J. 2002. The Spectral Features Analysis and Quantitative Remote Sensing Advances of Inland Water Quality Parameters [J], Geography and Territorial Research 2: 26-29.

[16] Mannheim, S.; Segl, K.; Heim, B.; Kaufmann, H. 2004. Monitoring of lake water quality using hyperspectral CHRIS-PROBA data. Proc. of the 2nd CHRIS/PROBA Workshop, 28- 30 April 2004, ESA/ESRIN, Frascati, Italy (ESA SP-578, July 2004).

[17] Morris, H. R.; Hyot, C. C.; Treado, P. J. 1994. Imaging Spectrometers for Fluorescence and Raman Microscopy: Acousto-Optic and Liquid Crystal Tunable Filters, Applied Optics (48): 857-866.

[18] Pellerin, B. A.; Bergamaschi, B. A.; Murdoch, P. S.; Downing, B. D.; Saraceno, J. F.; Aiken, G. R.; Striegl, R. G. 2011. The aquatic real-time monitoring network; in-situ optical sensors for monitoring the nation's water quality, U.S. Geological Survey Fact Sheet $3061: 2$. 
[19] Sader, E.; Abdallah, S.-A. 2013. Design of an optical water pollution sensor using a single-layer guided-mode resonance filter, Photonic Sensors, 1-7.

[20] Sepulcre-Cantó, G.; Zarco-Tejada, P. J.; Jiménez-Muñoz, J. C.; Sobrino, J. A.; Soriano, M. A.; Fereres, E.; . Pastor, M. 2007. Monitoring yield and fruit quality parameters in open-canopy tree crops under water stress. Implications for ASTER, Remote Sensing of Environment 107(3): 455-470. http://dx.doi.org/10.1016/j.rse.2006.09.014

[21] Sledzinska, I. 2012. The phenomenon of rotation of plane polarized light-akademic script. Warsaw University of Technology, Warsaw, Poland.

[22] Suresh, S. 2008. Visible to Near Infrared Hyperspectral Imaging: Visualizing the Neurological and Biological Nature of Ischemic Tissue. ProQuest,

[23] Raszewski, Z.; Olifierczuk, M.; Piecek, W. 2013. Advanced applications of liquid crystal materials - akademic script, Military University of Technology, Warsaw, Poland.

[24] VariSpec Liquid Crystal Tunable 2006. Filters User's Manual, Woburn (USA),Cambridge Research \& Instrumentation, Inc

[25] Zietek, B. 2011. Optoelectronics. Scientific Publishers Nicolaus Copernicus University, ISBN: 978-83-231-2746-8

[26] http://www.cri-inc.com - available: 16 December 2013

[27] http://www.jascoinc.com - available: 16 December 2013 